\title{
DETERMINAÇÃO DE HIDROCARBONETOS POLICÍCLICOS AROMÁTICOS (HPAS) EM GUARANÁ EM PÓ (Paullinia cupana) ${ }^{1}$
}

\author{
Mônica Cristiane Rojo CAMARGO, Sílvia Amélia Verdiani TFOUNI ${ }^{2, *}$, Sílvia Helena Pereira VITORINO ${ }^{3, *}$, \\ Thaís Feres MENEGÁRIO ${ }^{4}$, Maria Cecília de Figueiredo TOLEDO ${ }^{3}$
}

\section{RESUMO}

Hidrocarbonetos policíclicos aromáticos (HPAs) representam uma importante classe de carcinógenos químicos formados durante a combustão incompleta de material orgânico. Os HPAs ocorrem como contaminantes em diferentes tipos de alimentos, devido principalmente à poluição ambiental e alguns tipos de processamentos como a defumação, a secagem e a torrefação. Nos últimos anos, o consumo de guaraná vem aumentando, assim como a sua oferta no mercado. Durante o processamento das sementes para a obtenção dos produtos, a matéria-prima passa pelas etapas de secagem, torrefação e, em alguns casos, a defumação, processos estes que podem dar origem aos HPAs. Dessa forma, o presente estudo foi conduzido com o objetivo de avaliar a presença de cinco HPAs carcinogênicos: benzo(a)antraceno, benzo(b)fluoranteno, benzo(k)fluoranteno, benzo(a)pireno e dibenzo(a,h)antraceno em diferentes marcas de guaraná em pó disponíveis comercialmente. A determinação foi feita por Cromatografia Líquida de Alta Eficiência com Detecção por Fluorescência. Os HPAs foram detectados em $81 \%$ das amostras analisadas, com níveis variando de 0,05 a $8,04 \mu \mathrm{g} / \mathrm{kg}$. Os resultados indicam que o tipo de processamento utilizado durante a manufatura do guaraná em pó pode resultar na presença desses contaminantes no produto final.

Palavras-chave: hidrocarbonetos policíclicos aromáticos, HPAs, guaraná em pó, CLAE.

\section{SUMMARY}

DETEMINATION OF POLYCYCLIC AROMATIC HYDROCARBONS (PAHS) IN GUARANÁ POWDER (Paullinia cupana). Polycyclic aromatic hydrocarbons (PAHs) represent an important group of chemical carcinogens formed during incomplete combustion of organic matter. PAHs occur as contaminants in different types of food and the main sources of contamination are environmental pollution and food processing such as drying, roasting and smoking. In the last years, the consumption of guaraná products has increased, as well as their market offer. During processing, the seeds are dried and roasted and in some cases they are submitted to a smoking step, these procedures can originate PAHs. The objective of the present study was to determine the levels of five carcinogenic PAHs: benzo(a)anthracene, benzo(b)fluoranthene, benzo(k)fluoranthene, benzo(a)pyrene and dibenzo(a,h)anthracene in different brands of guaraná powder commercially available in Brazil. Determination was carried out by high performance liquid chromatography with fluorescence detection. PAHs were detected in $81 \%$ of the analysed samples with levels ranging from 0.05 to $8.04 \mu \mathrm{g} / \mathrm{kg}$. Results indicate that the processing used during guaraná powder production may result in the presence of these contaminants in the final product. Keywords: polycyclic aromatic hydrocarbons, PAHs, guaraná powder, HPLC.

\section{1 - INTRODUÇÃO}

Hidrocarbonetos policíclicos aromáticos (HPAs) são um grupo de compostos formados durante a combustão incompleta de material orgânico, como a queima de óleo ou madeira [1, $15,33]$. A quantidade e a composição dos HPAs produzidos dependem das condições de reação, temperatura e quantidade de ar [33]. A formação desses compostos é favorecida pela queima da matéria orgânica em temperaturas variando de 500 a $900^{\circ} \mathrm{C}$, principalmente acima de $700^{\circ} \mathrm{C}$ [2].

Os HPAs têm recebido muita atenção nos últimos anos devido ao fato de que muitos compostos desse grupo são potentes carcinógenos em animais experimentais [17,

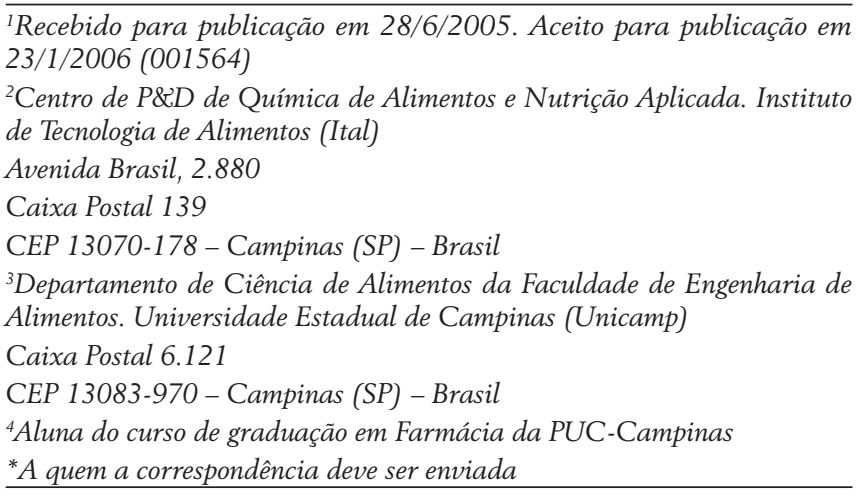

23/1/2006 (001564)

${ }^{2}$ Centro de P\&D de Química de Alimentos e Nutrição Aplicada. Instituto de Tecnologia de Alimentos (Ital)

Avenida Brasil, 2.880

Caixa Postal 139

CEP 13070-178-Campinas (SP) - Brasil

${ }^{3}$ Departamento de Ciência de Alimentos da Faculdade de Engenharia de Alimentos. Universidade Estadual de Campinas (Unicamp)

Caixa Postal 6.121

${ }^{4}$ Aluna do curso de graduação em Farmácia da PUC-Campinas

*A quem a correspondência deve ser enviada

34]. O Comitê Conjunto FAO/OMS de Peritos em Aditivos Alimentares (Jecfa) durante sua $64^{\mathrm{a}}$ reunião, após revisar e avaliar todas as informações toxicológicas relevantes, concluiu que dentro do grupo de HPAs, 13 compostos são claramente carcinogênicos e genotóxicos, inclusive os cinco selecionados para este estudo [35].

Provenientes, principalmente, de processamentos como defumação, secagem, torrefação, alguns tipos de cozimento, material de embalagem, assim como da poluição ambiental [2, 15,21 ], esses contaminantes ocorrem em diferentes grupos de alimentos e bebidas incluindo vegetais, frutas, carnes, óleos e gorduras, cereais e derivados, produtos lácteos, café, chá, entre outros $[8,9,10,11,12,15,16,22,24,25,27,28,32,36]$.

O guaranazeiro, planta de onde se obtém o guaraná, é nativo da Amazônia e pertencente à família das sapindáceas. O Brasil é praticamente o único produtor de guaraná do mundo, sendo que a maior parte é proveniente do Estado da Bahia, seguido por Amazonas e Mato Grosso [26]. O guaraná é comercializado em ramas (sementes torradas), seja para a exportação ou agroindustrialização. A partir das ramas podem ser obtidos o xarope (consumido diretamente como bebida energética ou usado para a produção industrial de refrigerantes gaseificados), o bastão (usado para ralar e obter o pó) ou o pó, que pode ser acondicionado em frascos, cápsulas gelatinosas ou saches [30]. 
A produção de ramas de guaraná no País é estimada em 4,3 mil ton por ano, sendo que $70 \%$ da produção é absorvida pela indústria de refrigerante (sob a forma de xarope) e os $30 \%$ restantes abastecem o mercado interno e externo, na forma de pó, xarope e bastão [30].

Durante o processamento dos frutos do guaraná para a obtenção dos produtos, a matéria-prima passa por diversas etapas, entre elas secagem, torrefação e, em alguns casos, defumação, processos estes que podem resultar na formação de HPAs [3].

No âmbito do Codex Alimentarius, a necessidade de estabelecimento de limites para HPAs em alimentos tem sido manifestada por inúmeros países em diferentes oportunidades, sendo este tema considerado prioritário dentro do Comitê do Codex para Aditivos Alimentares e Contaminantes (CCFAC). Em abril de 2005, este recomendou que sejam elaboradas estratégias de modo a minimizar a contaminação durante os processos de secagem e defumação de alimentos [4].

Entre os métodos utilizados para análise de HPAs em alimentos, a Cromatografia Líquida de Alta Eficiência tem sido amplamente utilizada em diferente matrizes [6, 7, $10,13,18,19]$.

Considerando-se a não disponibilidade de dados nacionais quanto à contaminação de produtos à base de guaraná por HPAs, o presente estudo foi conduzido com o objetivo de avaliar a presença de benzo(a)antraceno, benzo(b)fluoranteno, benzo(k)fluoranteno, benzo(a)pireno e dibenzo(a,h)antraceno em diferentes marcas de guaraná em pó disponíveis comercialmente.

\section{2 - MATERIAL E MÉTODOS}

\section{1 - Amostras}

Treze marcas de guaraná em pó foram selecionadas para a avaliação da contaminação por HPAs. Os produtos foram adquiridos no comércio das cidades de Campinas (SP) e Ribeirão Preto (SP), em três coletas distintas durante o ano de 2003. Um total de 39 amostras (13 marcas, três lotes de cada) foram analisadas quanto à presença de cinco HPAs: benzo(a)antraceno (B(a)A), benzo(b)fluoranteno $(\mathrm{B}(\mathrm{b}) \mathrm{F})$, benzo(k)fluoranteno $(\mathrm{B}(\mathrm{k}) \mathrm{F})$, benzo(a)pireno $(\mathrm{B}(\mathrm{a}) \mathrm{P})$ e dibenzo(a,h)antraceno (D(ah)A).

\section{2 - Solventes e reagentes}

Os padrões de B(a)A e D(ah)A foram adquiridos da Supelco Inc., enquanto que os demais foram obtidos da Aldrich Chemical Co. Os solventes e reagentes utilizados foram acetonitrila e metanol grau cromatográfico (Mallinckrodt Baker Inc.); cicloexano grau cromatográfico (Tedia Company Inc.); sulfato de sódio anidro, hidróxido de potássio e sílica gel 60 (70-230 mesh, ASTM) da Merck AS Chemical Industries. A água utilizada foi obtida por meio de um sistema de purificação Milli-Q (Millipore Co.).

\section{3 - Preparação e limpeza das amostras}

O método de extração utilizado foi baseado no procedimento descrito por HISCHENHUBER E STIJVE [13] para a determinação de benzo(a)pireno em café, com introdução de uma etapa de limpeza, de acordo com SPEER et al. [20]. Todas as amostras foram analisadas em duplicata.

\subsection{1 - Extração}

Pesou-se 20 g de amostra em um balão de fundo redondo de 500 mL. Adicionou-se 200 mL de KOH metanólico 1 M, e o material permaneceu sob refluxo por $40 \mathrm{~min}$. Em seguida, adicionou-se pelo condensador $200 \mathrm{~mL}$ de cicloexano permitindo-se uma ebulição de mais de $5 \mathrm{~min}$. Após atingir a temperatura ambiente, a solução foi filtrada em funil de Buchner e o material saponificado foi transferido para um funil de separação. Adicionou-se $200 \mathrm{~mL}$ de água e o funil foi agitado por aproximadamente 2 min. Após a separação das fases descartou-se a fase aquosa, lavou-se o extrato com três porções de água ( $100 \mathrm{~mL}$ ) e retirou-se a água residual com 2,5 g de sulfato de sódio anidro. A seguir, concentrou-se a solução a um volume final de aproximadamente $5 \mathrm{~mL}$ em evaporador rotativo à vácuo, com banho à temperatura de $40^{\circ} \mathrm{C}$.

Análises de controle sobre os reagentes (análise do branco) foram simultaneamente realizadas com cada série de amostras.

\subsection{2 - Limpeza em coluna de sílica gel}

Para a limpeza do extrato, foi utilizada uma coluna de vidro (21 x 1,1 cm d.i. e reservatório de $8 \times 3,1 \mathrm{~cm}$ d.i.) empacotada com 5 g de sílica gel 60 (70-230 mesh ASTM) e $40 \mathrm{~mL}$ de cicloexano, adicionando-se sulfato de sódio anidro no topo da mesma. A sílica gel utilizada foi desativada com 15\% de água, para evitar a degradação dos HPAs. O extrato foi aplicado à coluna e a fração, após ser eluída por gravidade com $85 \mathrm{~mL}$ de cicloexano, foi recolhida em um balão de fundo redondo de $250 \mathrm{~mL}$ e concentrada em um evaporador rotativo (banho a $40^{\circ} \mathrm{C}$ ), até aproximadamente $2 \mathrm{~mL}$. Após secagem sob corrente de nitrogênio, o resíduo foi suspendido em $2 \mathrm{~mL}$ de acetonitrila e submetido à análise cromatográfica.

\section{4 - Análise cromatográfica}

Os HPAs foram determinados por Cromatografia Líquida de Alta Eficiência (CLAE) com Detecção por Fluorescência. Foi utilizado um sistema cromatográfico Waters constituído por uma bomba quaternária modelo 600 , injetor automático modelo 717 degasser e detector de fluorescência modelo 474, programado nos comprimentos de onda de $290 \mathrm{~nm}$ (excitação) e $430 \mathrm{~nm}$ (emissão). Os dados foram registrados e analisados utilizando o software Millenium 32. O sistema também estava equipado com uma coluna cromatográfica C18 (Vydac $201 \mathrm{TP}$ ) de $25 \mathrm{~cm} \mathrm{x} \mathrm{4,6} \mathrm{mm} \mathrm{d.i.,} \mathrm{partículas}$ de $5 \mu \mathrm{m}$, termicamente estável a $30^{\circ} \mathrm{C}$. As amostras foram eluídas com fase móvel isocrática composta de acetonitrilaágua $(75: 25, \mathrm{v} / \mathrm{v})$, a uma vazão de $1,0 \mathrm{~mL} / \mathrm{min}$. O volume de injeção foi de $30 \mu \mathrm{L}$. 


\section{5 - Quantificação}

A quantificação foi feita por padronização externa. Foram realizadas injeções de $30 \mu \mathrm{L}$, em duplicata, das soluções-padrão de B(a)A, B(b)F, B(k)F, B(a)P e D(ah)A em sete concentrações na faixa de 0,1 a $100,0 \mathrm{ng} / \mathrm{mL}$. A identidade dos picos foi confirmada através da comparação dos tempos de retenção dos picos obtidos com os dos respectivos padrões e por adição dos padrões com sobreposição dos cromatogramas (co-Cromatografia).

\section{6 - Recuperação e limite de detecção}

Os testes de recuperação foram feitos adicionando-se quantidades conhecidas de cada HPA em amostras de pó de guaraná, em três diferentes concentrações variando entre 1 e $9,6 \mu \mathrm{g} / \mathrm{kg}$. As análises das amostras fortificadas, assim como dos controles, foram feitas em duplicata. As recuperações foram calculadas pela diferença entre a concentração dos compostos nas amostras fortificadas e não-fortificadas. Os resultados analíticos reportados não foram corrigidos em função da porcentagem de recuperação.

O limite de detecção dos HPAs foi estimado conforme proposto por TAYLOR [31].

\section{3 - RESULTADOS E DISCUSSÃO}

Os resultados dos ensaios de recuperação estão apresentados na Tabela 1 juntamente com os limites de detecção. Os valores médios de recuperação obtidos ficaram acima de $80 \%$, com um coeficiente de variação máximo de $10,7 \%$, dentro dos limites aceitos para análise de contaminantes em níveis de $\mu \mathrm{g} / \mathrm{kg}$ [14]. Estes resultados indicam que os procedimentos de extração e análise empregados foram adequados para a matriz analisada.

As Figuras 1 e 2 apresentam, respectivamente, cromatogramas típicos da solução dos padrões e cromatogramas obtidos para amostras de guaraná em pó.

Na Tabela 2, podem ser observadas as concentrações médias de HPAs determinadas nas amostras de guaraná em pó. Em $81 \%$ das amostras, pelo menos um dos cinco HPAs analisados estava presente, sendo que em $35 \%$ das amostras, todos os cinco HPAs em estudo foram detectados. Os teores médios para HPAs individuais se situaram na faixa de não detectado a 8,04 $\mu \mathrm{g} / \mathrm{kg}$. Benzo(a)antraceno, benzo(b)fluoranteno e benzo(a)pireno foram os hidrocarbonetos que ocorreram mais freqüentemente, em $76 \%$, 60\% e $60 \%$ das amostras, respectivamente. A marca $\mathrm{D}$ foi a que apresentou a maior contaminação média por HPAs, com um somatório de 13,95 $\mu \mathrm{g} / \mathrm{kg}$. A amostra com maior nível de HPAs somados foi aquela de um lote do guaraná em pó D contendo $36,3 \mu \mathrm{g} / \mathrm{kg}$.

Considerando-se a soma dos HPAs, é possível observar uma ampla faixa de variação nos níveis entre as diferentes marcas avaliadas $(0,05$ a $13,95 \mu \mathrm{g} / \mathrm{kg}$ ) (Tabela 2). Os valores médios determinados na marca $\mathrm{D}$, por exemplo, são quase 300 vezes maiores do que aqueles obtidos para a marca E. Essas variações também foram elevadas entre
TABELA 1 - Recuperação e limites de detecção para os diferentes HPAs em guaraná em pó

\begin{tabular}{lccccc}
\hline HPA & $\begin{array}{c}\text { Fortificação } \\
(\boldsymbol{\mu g} / \mathbf{k g})\end{array}$ & $\begin{array}{c}\text { Recuperação } \\
(\%)\end{array}$ & $\begin{array}{c}\text { Recuperação } \\
\text { média }(\%)\end{array}$ & $\begin{array}{c}\text { CV } \\
(\%)^{1}\end{array}$ & $\begin{array}{c}\text { Limite de } \\
\text { detecção } \\
(\boldsymbol{\mu g} / \mathbf{k g})\end{array}$ \\
\hline $\mathrm{B}(\mathrm{a}) \mathrm{A}$ & 1,2 & 99 & 95,5 & 5,9 & 0,04 \\
& 3 & 89 & & & \\
\hline $\mathrm{B}(\mathrm{b}) \mathrm{F}$ & 9,6 & 98,6 & & & \\
& 1 & 97,2 & 91,4 & 7,4 & 0,02 \\
& 2,5 & 84 & & & \\
\hline $\mathrm{B}(\mathrm{k}) \mathrm{F}$ & 8,2 & 92,9 & & & \\
& 1 & 78,6 & 83,5 & 10,7 & 0,02 \\
& 2,5 & 78 & & & \\
\hline $\mathrm{B}(\mathrm{a}) \mathrm{P}$ & 8,2 & 93,8 & & & \\
& 1,2 & 78,6 & 81,9 & 7,7 & 0,08 \\
& 3 & 78 & & & \\
\hline $\mathrm{D}($ ah)A & 9,5 & 89,2 & & & 0,21 \\
& 1 & 92,2 & 87,4 & 8,4 & \\
\hline${ }^{\mathrm{C}} \mathrm{CV}-$ coeficiente de variação & 91,1 & & & \\
\hline
\end{tabular}

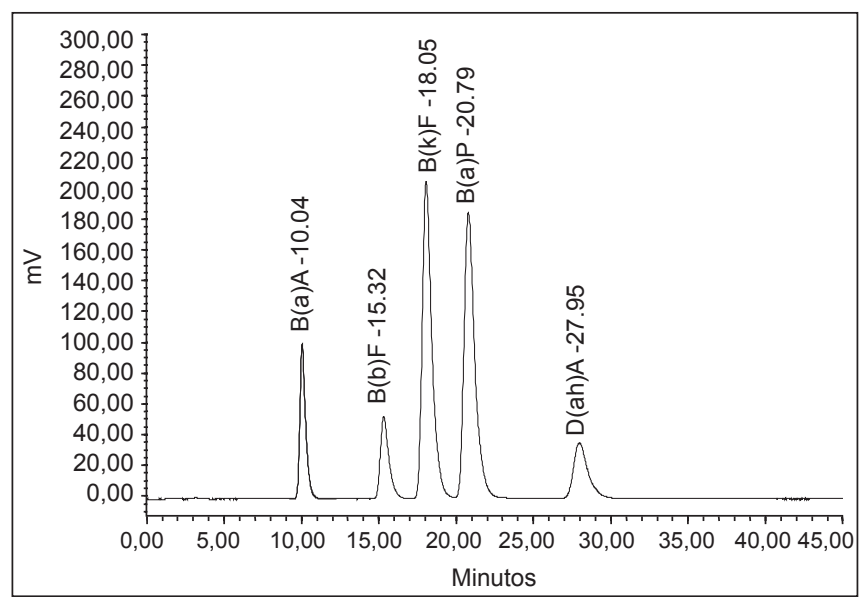

FIGURA 1 - Cromatograma por CLAE referente à solução-padrão de HPAs. Coluna C18 (Vydac 201 TP); fase móvel acetonitrila-água (75:25, v/v); vazão: $1 \mathrm{~mL} / \mathrm{min}$; volume injetado: $30 \mu \mathrm{L}$, detecção por fluorescência (excitação: 290 nm, emissão: $430 \mathrm{~nm}$ )

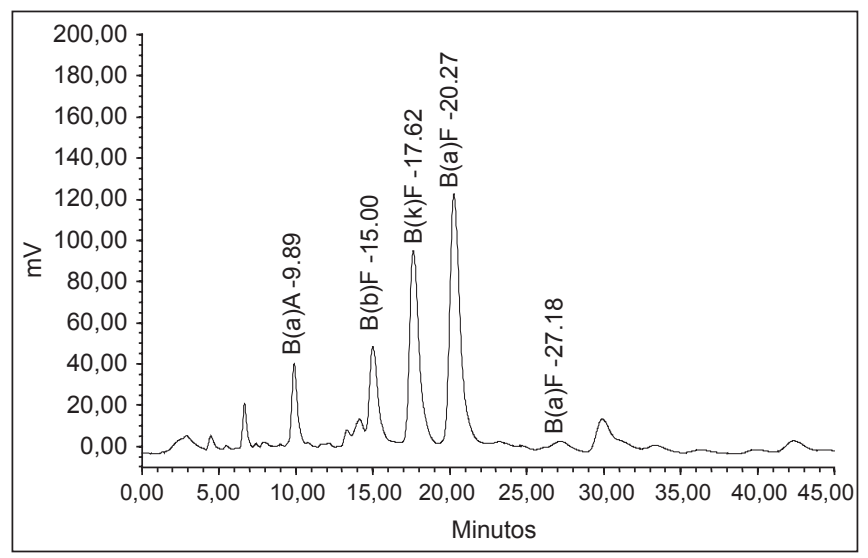

FIGURA 2 - Cromatograma por CLAE referente a uma amostra de guaraná em pó. Coluna C18 (Vydac 201 TP); fase móvel acetonitrilaágua (75:25, v/v); vazão: $1 \mathrm{~mL} / \mathrm{min}$; volume injetado: $30 \mu \mathrm{L}$, detecção por fluorescência (excitação: $290 \mathrm{~nm}$, emissão: $430 \mathrm{~nm}$ ) 
TABELA 2 - Teores médios de HPAs em amostras de guaraná em pó

\begin{tabular}{|c|c|c|c|c|c|c|}
\hline Amostra & \multicolumn{6}{|c|}{$\begin{array}{l}\text { Teores de HPAs }(\mu \mathbf{g} / \mathbf{k g})^{*} \\
\text { (faixa) }\end{array}$} \\
\hline $\bar{A}$ & 0,07 (nd- 0,22 ) & 0,01 (nd-0,04) & nd & 0,04 (nd-0,13) & nd & 0,13 (nd-0,26) \\
\hline B & $1,33(0,17-2,12)$ & $0,38(0,06-0,78)$ & 0,07 (nd-0,16) & $0,22(0,13-0,31)$ & $0,13($ nd- 0,4$)$ & $2,13(0,36-3,76)$ \\
\hline c & $1,03(0,37-2,28)$ & $0,82(0,19-2,06)$ & 0,31 (nd-0,86) & $0,82(0,23-1,97)$ & 0,15 (nd-0,45) & $3,13(0,8-7,62)$ \\
\hline D & $3,72$ (nd- 9,35$)$ & $3,84(\mathrm{nd}-10,07)$ & $2,00(n d-5,34)$ & $3,69($ nd- $-9,65)$ & 0,71 (nd-1,9) & 13,95 (nd-36,3) \\
\hline E & $0,05$ (nd- 0,14$)$ & nd & nd & nd & nd & 0,05 (nd-0,14) \\
\hline H & $3,36(0,44-9,11)$ & $2,24(0,09-6,22)$ & 1,06 (nd-3,03) & $2,22(0,11-6,28)$ & 0,34 (nd-1,01) & $9,22(0,64-25,64)$ \\
\hline I & $1,17(n d-1,91)$ & $1,06(n d-1,74)$ & $0,45$ (nd- 0,74$)$ & $0,74(\mathrm{nd}-1,16)$ & nd & $3,42(\mathrm{nd}-5,56)$ \\
\hline $\mathbf{J}$ & 2,54 (nd-4,36) & $1,91(\mathrm{nd}-2,9)$ & $0,75(n d-1,14)$ & $1,63(\mathrm{nd}-2,5)$ & 0,29 (nd-0,43) & $7,12($ nd-11,25) \\
\hline K & $3,18(2,57-3,55)$ & $2,54(1,43-3,63)$ & $1,07(0,6-1,5)$ & $2,15(1,12-3,21)$ & 0,55 (nd-0,95) & $9,49(5,72-12,84)$ \\
\hline L & $8,04(5,98-10,1)$ & $1,39(1,08-1,71)$ & $0,4(0,15-0,65)$ & $1,1(0,86-1,34)$ & nd & $10,93(9,68-12,18)$ \\
\hline M & $2,34(1,05-3,82)$ & $2,12(0,61-3,74)$ & $0,89(0,23-1,5)$ & $1,83(0,55-3,01)$ & 0,34 (nd-0,66) & $7,53(2,44-12,73)$ \\
\hline
\end{tabular}

*Média de três lotes. nd - não detectado

diferentes lotes da mesma marca, o que pode ser observado nas marcas $\mathrm{D}, \mathrm{F}$ e $\mathrm{H}$, que apresentaram resultados desde não detectado até 36,3 e $22,77 \mu \mathrm{g} / \mathrm{kg}$ (para D e F, respectivamente) e 0,64 a $25,64 \mu \mathrm{g} / \mathrm{kg}$ para a marca $\mathrm{H}$.

Essa ampla faixa de concentração encontrada resulta, provavelmente, das diferentes formas de processamento a que o guaraná é submetido para a obtenção do produto final. As sementes são secas e torradas e comercializadas em ramas, sendo que em alguns casos podem passar também pelo processo de defumação. A grande variação entre lotes de uma mesma marca, por sua vez, deve-se, provavelmente, ao fato de que muitas empresas que comercializam o guaraná em pó não são responsáveis pelo processamento do produto. As empresas, muitas vezes, adquirem subprodutos como a rama e o bastão ou até mesmo o próprio pó de diferentes fornecedores, finalizam o processamento e embalam sob uma determinada marca comercial. Muitas vezes, o processamento do produto nos fornecedores chega a ser artesanal, com pouco ou nenhum controle nas diferentes etapas de processamento.

Como não existem na literatura dados sobre a presença de HPAs em guaraná em pó, há dificuldade em avaliar os níveis de contaminação encontrados. Desta forma, utilizando-se o pó de café para fins comparativos, observa-se que a quantidade média obtida para o somatório de HPAs presentes nas diferentes marcas de guaraná em pó pode ser até quatro vezes maior do que aquela encontrada no pó de café (3,15 $\mu \mathrm{g} / \mathrm{kg})$ [6].

Estudos anteriores já haviam relacionado o processo de secagem de folhas e grãos como fator importante na formação de HPAs e conseqüente contaminação de produtos finais como no caso de óleos vegetais [5,29], café, chá [6, 13, 20,] e chimarrão [23].

\section{4 - CONCLUSÕES}

O guaraná em pó é considerado pelo consumidor em geral como um produto natural e, portanto, isento de riscos à saúde. No entanto, os resultados apresentados mostraram que essa afirmativa pode não ser totalmente verdadeira em função da presença de HPAs nesse tipo de produto. O processamento utilizado na fabricação do guaraná em pó pode gerar altos níveis de HPAs, o que vem a ser preocupante principalmente entre consumidores freqüentes.

Desta forma, o presente estudo evidencia a importância de se elaborar medidas de intervenção para minimizar a formação e posterior contaminação do guaraná em pó por HPAs durante o processamento das sementes para a obtenção do produto final.

\section{5 - REFERÊNCIAS BIBLIOGRÁFICAS}

[1] ATSDR, 1996, Polycyclic aromatic hydrocarbons (PAHs). Agency for Toxic Substances and Disease Registry, Department of Health and Human Services, Public Health Service, Atlanta, GA, USA. Disponível em < http://www.atsdr. cdc.gov:8080/tfacts69.html>. Acesso em: 20 abr. 2005.

[2] BARTLE, K.D. Analysis and occurrence of polycyclic aromatic hydrocarbons in food. In: CREASER, C. \& PURCHASE, R. (Eds) Food contaminants: sources and surveillance. Cambridge, The Royal Society of Chemistry, cap. 3, p. 41-60, 1991.

[3] BRASIL. Decreto no 87,105, de 19 de abril de 1982. Normas de identidade, qualidade, embalagem, armazenamento e transporte do guaraná em grão, em bastão e em pó. Diário Oficial da União. Brasília (DF).

[4] CAC - CODEX ALIMENTARIUS COMITION. CX/FAC/05/37/4 - Action required as a result of changes in acceptable daily intake (ADI) status and other toxicological recommendations. The Hague, CAC, 6 p., 2005.

[5] CAMARGO, M.S.F.O.; TOLEDO, M.C.F. Efeito do processamento na contaminação de óleo refinado de milho por benzo(a)pireno. Brazilian Journal of Food Technology, v. 1, n. 1,2, p. 97-106, 1998.

[6] CAMARGO, M.C.R.; TOLEDO, M.C.F. Chá-mate e café como fontes de hidrocarbonetos policíclicos aromáticos (HPAs) na dieta da população de Campinas. Ciência e Tecnologia de Alimentos, v. 22, n. 1, p. 49-53, 2002.

[7] CAMARGO, M.C.R.; TOLEDO, M.C.F. Avaliação da contaminação de diferentes grupos de alimentos por hidrocarbonetos policíclicos aromáticos. Brazilian Journal of Food Technology, v. 5, n. 76, p. 19-26, 2002.

[8] DENNIS, M.J.; MASSEY, R.C.; McWEENY, D.J.; KNOWLES, M.E. Analysis of polycyclic aromatic hydrocarbons in UK total diets. Food Chemical Toxicology, v. 21, n. 5 , p. 569-574, 1983. 
[9] DE VOS, R.H.; VAN DOKKUM, W.; SCHOUTEN, A.; JONG-BERKHOUT, P. Polycyclic aromatic hydrocarbons in Dutch total diet samples (1984-1986). Food Chemical Toxicology, v. 28, n. 4, p. 263-268, 1990.

[10] GARCIA-FALCÓN, M.S.; GONZÁLEZ-AMIGO, S.; LAGEYUSTY, M.A. \& SIMAL-LOZANO, J. Determination of benzo(a)pyrene in some Spanish commercial smoked products by HPLC-FL. Food Additives and Contaminants, v. 16, n. 1, p. 9-14, 1999.

[11] GOMMA, E. A.; GRAY, J.I.; RABIE, S.; LOPEZ-BOTE, C.; BOOREN, A.M. Polycyclic aromatic hydrocarbons in smoked food product and commercial liquid smoke flavourings. Food Additives and Contaminants, v. 10, n. 5, p. 503-521, 1993.

[12] GREENBERG, A.; CHE-HAN, H.; ROTHMAN, N.; STRICKLAND, P.T. PAH profiles of charbroiled hamburgers: pyrene/B(a)P ratios and presence of reactive PAH. Polycyclic Aromatic Compounds, v. 3, n. 2, p. 101-110, 1993.

[13] HISCHENHUBER, C.; STIJVE, T. Determination of benzo(a)pyrene in roasted coffee and coffee brews by HPLC with fluorescence detection. Zeitschrift fur Lebensmittelkunde und Lebensmittelrecht, v. 83, n. 1, p. 1-4, 1987.

[14] HORWITZ, W.; KAMPS, L.R.; BOYER, K.W. Quality Assurance in the analyses of foods for trace constituents. Journal of the Association of Analytycal Chemistry, v. 63, n. 6, p. 1.344-1.354, 1980.

[15] HOWARD, J. W.; FAZIO, T. Review of polycyclic aromatic hydrocarbons in foods. Journal of Association of Official Analytical Chemists, v. 63, n. 5, p. 1.077-1.104, 1980.

[16] HUSAIN, A.; NAEEMI, E.; DASHTI, B.; Al-OMIRAH, H.; Al-ZENKI, S. Polycyclic aromatic hydrocarbons in food products originating from locally rared animals in Kuwait. Food Additives and Contaminants, v. 14, n. 3, p. 295-299. 1997.

[17] IARC - INTERNATIONAL AGENCY FOR RESEARCH OF CANCER. Polynuclear aromatic compounds. IARC Monographs on the evaluation of the carcinogenic risk of chemical to humans. Part 1, Chemical, Environmental and Experimental Data, v. 32, Dec., Lyon, France, IARC, 1983.

[18] KISHIKAWA, N., WADA, M., KURODA, N., AKIYAMA, S., \& NAKASHIMA, K. Determination of polycyclic aromatic hydrocarbons in milk samples by high-performance liquid chromatography with fluorescence detection. Journal of Chromatography B, v. 789, p. 257-264, 2003.

[19] LAGE-YUSTY, M.A. \& CORITZO-DAVIÑA, J.L. Supercritical fluid extraction and high-performance liquid chromatography-fluorescence detection method for polycyclic aromatic hydrocarbons investigation in vegetable oil. Food Control, v. 16, n. 1, p. 59-64, 2005.

[20] LARSSON, B.K. Polycyclic aromatic hydrocarbons in Swedish foods - aspects on analysis, occurrence and intake. Doctoral thesis, Swedish University of Agricultural Sciences, 1986.

[21] LO, M.; SANDI, E. Polycyclic aromatic hydrocarbons (polynuclears) in foods. Residue Review, v. 69, p. 35-56, 1978.

[22] LODOVICI, M.; DOLARA, P.;CASALINI, C.; CIAPPELLANO, S; TESTOLINI, G. Polycyclic aromatic hydrocarbon contamination in the Italian food. Food Additives and Contaminants, v. 12, n. 5, p. 703-713, 1995.

[23] MACHADO, L.S.; BERNASIUK, C.; SCHENKEL, E.; NOLL,
I.B.; TOLEDO, M.C.F. Determinação de hidrocarbonetos poliaromáticos em erva mate. II Simpósio Latino-americano de Ciência de Alimentos: progresso e ação para o ano 2000. Campinas, SP, 11-14 nov. 1997.

[24] NOLL, I.B.; TOLEDO, M.C.F. Benzo(a)pireno em carnes defumadas pelos processos caseiro e industrial. Revista Brasileira de Toxicologia, v. 10, n. 1, p. 19-23, 1997a.

[25] NOLL, I.B.; TOLEDO, M.C.F. Determinacion de benzo(a)pireno en asados tipo churrasco en Brasil. Información Tecnológica, v. 8, n. 2, p. 177-181, 1997b.

[26] PINTO, M.C.R. Guaraná: alguns aspectos da produção e da comercialização. Revista de Política Agrícola, v. 7, n. 1, 1998.

[27] PUPIN, A.M.; TOLEDO, M.C.F. Benzo(a)pyrene in Brazilian vegetables oils. Food Additives and Contaminants, v. 13, n. 6, p. 639-646, 1996.

[28] PUPIN, A.M.; TOLEDO, M.C.F. Benzo(a)pyrene in olive oils on the Brazilian market. Food Chemistry, v. 55, n. 2, p. 185-188, 1996.

[29] SPEER, K.; STEEG, E.; HORSTMANN, P.; KUHN, T.; MONTAG, A. Determination and distribution of polycyclic aromatic hydrocarbons in native vegetable oils, smoked fish products mussels and oysters, and bream from the river Elbe. Journal of High Resolution Chromatography, v. 13, p. 104-111, 1990.

[30] SUFRAMA - SUPERINTENDÊNCIA DA ZONA FRANCA DE MANAUS. Potencialidades regionais, estudo da viabilidade econômica, guaraná. Manaus, Suframa, 2003.

[31] TAYLOR, J.K. Quality assurance of chemical measurements. Lewis Publishers Inc., Chelsea, Michigan, chapter 9, p. 79, 1987.

[32] TOLEDO, M.C.F.; CAMARGO, M.S.F.O. Benzo(a)pireno em óleos de milho produzidos e comercializados no Brasil. Ciência e Tecnologia de Alimentos, v. 18, n. 1, p. 73-76, 1998.

[33] VAESSEN, H.A.M.G.; JEKEL, A.A.; WILBERS, A.A.M.M. Dietary intake of polycyclic aromatic hydrocabons. Toxicological and Environmental Chemistry, v. 16, p. 281-294, 1988.

[34] VAN SCHOOTEN, F.J.; VAN LEEUWEN, F.E.; HILLEBRAND, M.J.X.; RIJKE, M.E.; HART, A.A.M.; VAN VEEN, H.G.; OOSTERINK, S.; KRIEK, E. Determination of benzo(a)pyrene diol epoxide-DNA adducts in white blood cell DNA from coke-oven workers: the impact of smoking. Journal of the National Cancer Institute, v. 82, n. 11, Jun., p. 927-933, 1990.

[35] WHO - WORLD HEALTH ORGANIZATION. Summary and conclusions of the sixty-fourth meeting of the joint FAO/WHO Expert Committee on Food Additives. Rome: WHO, 47 p., 2005.

[36] YABIKU, H.Y.; MARTINS, M.; TAKAHASHI, M.Y. Levels of benzo(a)pyrene and others polycyclic aromatic hydrocabons in liquid smoke flavour and some smoked foods. Food Additives and Contaminants, v. 10, n. 4, p. 339-405, 1993.

\section{6 - AGRADECIMENTOS}

À Coordenação de Aperfeiçoamento de Pessoal de Nível Superior (Capes) e ao Conselho Nacional de Desenvolvimento Científico e Tecnológico (CNPq) pela concessão de auxílio financeiro à pesquisa. 